Full Length Article

\title{
Ethnobotanical Profile of Weed Flora of District Charsadda, Khyber Pakhtunkhwa
}

\author{
Muhammad Nauman Khan ${ }^{1, *}$, Abdul Razzaq², Fazal Hadi1 ${ }^{1}$, Naushad Khan ${ }^{1}$, Abdul Basit ${ }^{1}$, Farmanullah Jan ${ }^{1}$ and \\ Nasir Khan ${ }^{3}$ \\ 1Department of Botany, University of Peshawar, KP, Pakistan \\ ${ }^{2}$ Centre of Plant Biodiversity, University of Peshawar, KP, Pakistan \\ ${ }^{3}$ Department of Botany, Abdul Wali Khan University, Mardan, KP, Pakistan
}

\section{ABSTRACT}

District Charsadda is a very important center of plant biodiversity in the central plain of Peshawar valley, Pakistan. The present study was carried out during March 2015 to April 2016 to investigate the ethnobotanical profile of common weed flora present in district Charsadda, KP, Pakistan. The study revealed that there were 40 weed species belonging to 21 families. Among them 25 weeds were annual herb, 9 weeds were perennial herb, three were annual grass, one was climbing herb, one was the parasitic weed, and one was rhizomatic grass. The dominant families were Asteraceae, Fabaceae and Poaceae having 5 species (12.5\%) each followed by Ranunculaceae 3 species (7.5\%). Plants were systematically arranged into botanical names, local names, families, habit, habitat, partly used, flowering periods, locality and ethnobotanical uses. The main aim of the study is the documentation and ethnobotanical information of the weed flora growing in the area.

Keywords:

Ethnobotany, Weed species, Local uses,

Annual or Perennial, Wheat, Maize and Garlic

Crops, District Charsadda, Khyber

Pakhtunkhwa.

Cite this article: Khan MN, Razzaq A, Hadi F, Khan N, Basit A, Jan F, Khan N. Ethnobotanical profile of weed flora of district Charsadda, Khyber Pakhtunkhwa. RADS J. Biol. Res. Appl. Sci. 2018; 9(1): 14-23.

This is an Open Access article distributed under the terms of the Creative Commons Attribution License (http://creativecommons.org/licenses/by/4.0), which permits unrestricted use, distribution, and reproduction in any medium, provided the original work is properly cited.

\section{INTRODUCTION}

Charsadda derives its name from its headquarters town. At the time of Alexander's invasion Charsadda was known as Pushkalavati (The Lotus city). Charsadda district lies in the central plain of Peshawar valley between $34-03$ ' to 34$28^{\prime}$ North Latitude and 71-28' to 71-33' East Longitudes with area of is 996 square kilometres. It is bounded by Malakand district on the north, Mardan district on the east, Nowshera and Peshawar districts on the south and
Mohmand Agency on the west, Anonymous ${ }^{1}$. The plain of Charsadda district is very fertile. The mean maximum and minimum temperatures, Precipitation and humidity recorded at Charsadda as shown in Table 1. Major crops of the area are Tobacco, Sugarcane, Sugar beet, Wheat and Maize while major vegetables are Potato, Tomato, Cabbage, Brinjals and Spinach and major fruits are Apricot, Citrus, Plum, Strawberry and Pears. 
Table 1: Showing mean maximum and minimum temperature, rain and humidity.

\section{MONTHLY MEAN MAX TEMP. $\left({ }^{\circ} \mathrm{C}\right)$}

\begin{tabular}{lllllllllllll}
\hline YEAR & JAN & FEB & MAR & APR & MAY & JUN & JUL & AUG & SEP & OCT & NOV & DEC \\
\hline $\mathbf{2 0 1 4}$ & 20.7 & 20.1 & 22.6 & 30.0 & 34.8 & 41.2 & 37.6 & 37.0 & 35.5 & 29.9 & 25.5 & 20.8 \\
\hline $\mathbf{2 0 1 5}$ & 19.5 & 21.3 & 23.8 & 30.4 & 35.4 & 38.5 & 35.9 & 34.8 & 34.3 & 30.2 & 23.5 & Nil \\
\hline
\end{tabular}

MONTHLY MEAN MIN TEMP. $\left({ }^{\circ} \mathrm{C}\right)$

\begin{tabular}{lllllllllllll}
\hline YEAR & JAN & FEB & MAR & APR & MAY & JUN & JUL & AUG & SEP & OCT & NOV & DEC \\
\hline $\mathbf{2 0 1 4}$ & 2.9 & 6.1 & 10.2 & 15.5 & 20.1 & 24.6 & 26.4 & 25.9 & 23.7 & 18.1 & 9.0 & 3.8 \\
\hline $\mathbf{2 0 1 5}$ & 4.3 & 9.0 & 11.5 & 17.5 & 21.7 & 24.7 & 26.9 & 25.7 & 21.1 & 16.7 & 10.2 & Nil \\
\hline
\end{tabular}

MONTHLY TOTAL RAIN (MM) [-1=TRACE]

\begin{tabular}{lllllllllllll}
\hline YEAR & JAN & FEB & MAR & APR & MAY & JUN & JUL & AUG & SEP & OCT & NOV & DEC \\
\hline $\mathbf{2 0 1 4}$ & 5.2 & 40.6 & 119.7 & 54.8 & 16.6 & 27.8 & 48.4 & 61.0 & 15.0 & 43.2 & 1.2 & 0.0 \\
\hline $\mathbf{2 0 1 5}$ & 33.7 & 70.1 & 120.3 & 114.1 & 39.2 & 0.0 & 119.0 & 158.4 & 53.3 & 52.0 & 29.6 & Nil
\end{tabular}

MONTHLY MEAN HUMIDITY (\%) AT 0800 AM

\begin{tabular}{lllllllllllll}
\hline YEAR & JAN & FEB & MAR & APR & MAY & JUN & JUL & AUG & SEP & OCT & NOV & DEC \\
\hline $\mathbf{2 0 1 4}$ & 81 & 84 & 86 & 71 & 63 & 57 & 76 & 78 & 75 & 86 & 80 & 82 \\
\hline $\mathbf{2 0 1 5}$ & 84 & 83 & 85 & 81.1 & 64 & 63 & 82 & 85 & 79 & 87 & 86 & Nil \\
\hline
\end{tabular}

MONTHLY MEAN HUMIDITY (\%) AT 0500 PM

\begin{tabular}{lllllllllllll}
\hline YEAR & JAN & FEB & MAR & APR & MAY & JUN & JUL & AUG & SEP & OCT & NOV & DEC \\
\hline $\mathbf{2 0 1 4}$ & 52 & 58 & 86 & 38 & 37 & 33 & 66 & 49 & 51 & 62 & 53 & 54 \\
\hline 2015 & 54 & 54 & 62 & 50 & 42 & 42 & 62 & 67 & 56 & 55 & 60 & Nil \\
\hline
\end{tabular}

\section{Source: Pakistan Meteorological Department}

Ethnobotany is a biological and economic cultural relationship between people and plants of an area in which they exist. Indigenous knowledge of plants is as old as civilization but the term ethno botany was used for the first time by an American botanist John. W. Harsh Berger in 1896 , to study plants used by primitive and indigenous communities ${ }^{2}$. Weeds are considered as unwanted plants. They give tough time to any specific crop in which it occurs. Its diversity and distribution varies from crop to crop. Such vegetation is found abundant in cultivated fields of great economic and ecological importance ${ }^{3}$. Sher et al. stated that weeds are undesirable on account of their competitive and allelopathic behavior and providing habitats for harmful organisms ${ }^{4}$. The yield per acre of wheat can be increased by agronomic practices including weed control. However, the authentic identification and distribution has always been a pre-requisite for weed management. Ali \& coworkers defined flora as all plant species in any specific geographic region, which are 
characteristic of a geological period or that inhabit a particular ecosystem ${ }^{5}$. The flora includes the number of species. The principal object of a Flora is to afford the means of determining any plant growing in the area circumscribed. A good Flora is one that provides work for correct identification of plant and their utilization could be taken on scientific and systematic basis. Medicinally important plants are necessary for the production of the various drugs and curing diseases. People use 26 species of the vascular plants of the Mianwali district used for different purposes i-e as a medicine, as furniture and agricultural implements and as a food ${ }^{6}$. Bhushan et al. stated that the study of direct relationship between man and plants ${ }^{7}$. The villagers have their own remedies for medicinal treatment by using various plants or plant products present in their vicinity. Ibrar et al. estimated that there were 37 fuel species, 37 fodder species, 31 medicinal species, 18 edible species and 10 vegetables species which are used in our daily life ${ }^{8}$. Valuable economic and ethno medicinal flora of the area are decreasing. Sustainable utilization and conservation of the flora of the area is strictly recommended ${ }^{9}$. Ali et al. reported 65 species belonging to 35 families, from the Valley coined to the ethno medical domain of the Tirat Valley, Swat District, Pakistan ${ }^{10}$. Pandey et al. suggested that plant part used by the locals are: the whole plants $(41.5 \%)$ followed by seed, 4 leaves, 11 flowers, 9 roots, 4 barks, 7 gums, 4 stems, 1 fruit, 7 resins and 1 wood, stem and acorns ${ }^{11}$. The uses of ethnomedicinal plants should be confirmed the experimental and phytochemical studies to increase the safety and effectiveness of these plants and witness to develop modern drug development ${ }^{12}$. Zabeehullah et al. explored traditional knowledge of ethno medicinal plants of District Charsadda, KP Pakistan ${ }^{13}$. The current studies focused on the information regarding indigenous uses of plants for medicinal purposes. 60 species of plant included 34 families have been recorded. During investigation, the data revealed that $56 \%$ of folk medicinal knowledge comes from people above the age of 60 years while $26 \%$ comes from between ages of $25-59$ years. While $13 \%$ comes from between age of $10-24$. Children of ages $8-10$ are below got $7 \%$ of medicinal plants traditionally knowledge.

\section{MATERIALS AND METHODS}

The study was carried out in the flowering seasons during 2015-2016 and plant specimens were collected from different crops field Viz Wheat, Maize and sugar cane fields and were kept in presser, dried for a week and identified with the help of flora of Pakistan ${ }^{14-16}$. The ethnobotanical information from the local inhabitants were gathered (including Hakeem, farmer, shopkeepers and old age people of the area) through discussion method and interviews. Geological information were obtained from the office of Geological Survey of Pakistan, Peshawar. Population data were obtained from census report (1998), from Department of Geography, University of Peshawar.

\section{RESULTS AND DISCUSSION}

The current study is based on economic importance of weed flora of district Charsadda, Pakistan. The study revealed a total of 40 species belonging to 21 families. The leading families are Asteraceae, Fabaceae and Poaceae 5 species (12.5\%) each followed by Ranunculaceae 3 species (7.5\%). Amaranthaceae, Apiaceae, Brassicaceae, Plantaginaceae, Caryophyllaceae 2 species (5.4\%) each while the remaining 14 families are monophilitic and comprised on single species (2.5\%) each (Table 2).

Table 2: Summary of percentage of different families.

\begin{tabular}{|c|c|c|c|}
\hline S.No. & Families & $\begin{array}{c}\text { No. of } \\
\text { species }\end{array}$ & Percentage \% \\
\hline 1. & Asteraceae & 5 & $12.5 \%$ \\
\hline 2. & Fabaceae & 5 & $12.5 \%$ \\
\hline 3. & Poaceae & 5 & $12.5 \%$ \\
\hline 4. & Ranunculaceae & 3 & $7.5 \%$ \\
\hline 5. & Amaranthaceae & 2 & $5 \%$ \\
\hline 6. & Apiaceae & 2 & $5 \%$ \\
\hline 7. & Brassicaceae & 2 & $5 \%$ \\
\hline 8. & Caryophyllaceae & 2 & $5 \%$ \\
\hline 9. & Plantaginaceae & 2 & $5 \%$ \\
\hline 10. & Boraginaceae & 1 & $2.5 \%$ \\
\hline 11. & Cannabinaceae & 1 & $2.5 \%$ \\
\hline 12. & Chenopodiaceae & 1 & $2.5 \%$ \\
\hline 13. & Convululaceae & 1 & $2.5 \%$ \\
\hline 14. & Cuscutaceae & 1 & $2.5 \%$ \\
\hline 15. & Euphorbiaceae & 1 & $2.5 \%$ \\
\hline 16. & Fumariaceae & 1 & $2.5 \%$ \\
\hline 17. & Lamiaceae & 1 & $2.5 \%$ \\
\hline 18. & Oxilidaceae & 1 & $2.5 \%$ \\
\hline 19. & Papaveraceae & 1 & $2.5 \%$ \\
\hline 20. & Polygonaceae & 1 & $2.5 \%$ \\
\hline \multirow[t]{2}{*}{21.} & Solanaceae & 1 & $2.5 \%$ \\
\hline & Total & 40 & $100 \%$ \\
\hline
\end{tabular}


Among them 25 species are annual herb, 9 species were perennial herb, 3 is annual grass, 1 is annual climbing herb, 1 is parasitic weed and one is rhizomitic grass (Table 3).
Botanical names followed by local name. Plants were systematically arranged into botanical name, local name, habit, crops field, habitat, part used, ethnobotanical uses, flowering periods and their localities are shown in Table 4.

Table 3: Summary of percentage habit class.

\begin{tabular}{cccc}
\hline S.No. & Habit class & No. of species & Percentage $\%$ \\
\hline 1. & Annual herb & 25 & $\mathbf{6 2 . 5 \%}$ \\
\hline 2. & Perennial herb & 9 & $\mathbf{2 2 . 5 \%}$ \\
\hline 3. & Annual grass & 3 & $\mathbf{7 . 5 \%}$ \\
\hline 4. & Parasitic weed & 1 & $2.5 \%$ \\
\hline 5. & Rhizomatic grass & 1 & $\mathbf{2 . 5 \%}$ \\
\hline 6. & Climbing herb & 1 & $\mathbf{2 . 5 \%}$ \\
\hline \multicolumn{4}{r}{} \\
\hline
\end{tabular}

Table 4: Checklist of ethnobotanical profile of common weed flora growing in district Charsadda, KP, Pakistan.

\begin{tabular}{|c|c|c|c|c|c|c|c|c|c|}
\hline S.No. & Family/ Botanical Name & Local Name & Habit & $\begin{array}{l}\text { Crop } \\
\text { field }\end{array}$ & Habitat & $\begin{array}{l}\text { Part } \\
\text { used }\end{array}$ & $\begin{array}{l}\text { Ethnobotanical } \\
\text { Uses }\end{array}$ & $\begin{array}{l}\text { Flowering } \\
\text { periods }\end{array}$ & Locality \\
\hline \multirow[t]{3}{*}{1.} & \multicolumn{9}{|l|}{ Amaranthaceae } \\
\hline & Achyranthus aspera L. & Kotheri & $\begin{array}{l}\text { Annual } \\
\text { herb }\end{array}$ & $\begin{array}{l}\text { Maize } \\
\text { field }\end{array}$ & $\begin{array}{l}\text { Cultivated } \\
\text { and } \\
\text { waste } \\
\text { fields }\end{array}$ & $\begin{array}{l}\text { Whole } \\
\text { plant }\end{array}$ & $\begin{array}{l}\text { Diuretic, } \\
\text { toothache, } \\
\text { cough, } \\
\text { asthama, } \\
\text { astringent, } \\
\text { laxative and } \\
\text { also used for } \\
\text { removal of } \\
\text { kidney stone. }\end{array}$ & $\begin{array}{l}\text { June- } \\
\text { August }\end{array}$ & $\begin{array}{l}\text { Through out } \\
\text { Charsadda }\end{array}$ \\
\hline & Amaranthus viridus $\mathrm{L}$. & Gankhar & $\begin{array}{l}\text { Perennial } \\
\text { herb }\end{array}$ & $\begin{array}{l}\text { Wheat } \\
\text { and } \\
\text { Garlic } \\
\text { field }\end{array}$ & $\begin{array}{l}\text { Cultivated } \\
\text { and } \\
\text { waste } \\
\text { fields }\end{array}$ & $\begin{array}{l}\text { Leaves, } \\
\text { stem and } \\
\text { seeds }\end{array}$ & $\begin{array}{l}\text { Vegetable and } \\
\text { commonly used } \\
\text { as fodder }\end{array}$ & April-June & $\begin{array}{l}\text { Through out } \\
\text { Charsadda }\end{array}$ \\
\hline \multirow[t]{3}{*}{2.} & \multicolumn{9}{|l|}{ Apiaceae } \\
\hline & Scandix pectin veneris $L$. & Kali ziri & $\begin{array}{l}\text { Annual } \\
\text { herb }\end{array}$ & $\begin{array}{l}\text { Wheat } \\
\text { field }\end{array}$ & $\begin{array}{l}\text { Cultivated } \\
\text { fields }\end{array}$ & $\begin{array}{l}\text { Whole } \\
\text { plant }\end{array}$ & $\begin{array}{l}\text { Grazed as } \\
\text { fodder. }\end{array}$ & $\begin{array}{l}\text { March- } \\
\text { May }\end{array}$ & $\begin{array}{l}\text { Shabqadar, Tangi, } \\
\text { Umarzai }\end{array}$ \\
\hline & $\begin{array}{l}\text { Torilis leptophylla (L.) } \\
\text { reichenb.f. }\end{array}$ & $\begin{array}{l}\text { Zangali } \\
\text { gajer }\end{array}$ & $\begin{array}{l}\text { Annual } \\
\text { herb }\end{array}$ & $\begin{array}{l}\text { Wheat } \\
\text { field }\end{array}$ & $\begin{array}{l}\text { Cultivated } \\
\text { fields }\end{array}$ & $\begin{array}{l}\text { Whole } \\
\text { plant }\end{array}$ & $\begin{array}{l}\text { Commonly used } \\
\text { as fodder }\end{array}$ & Feb-April & $\begin{array}{l}\text { Shabqadar, Tangi, } \\
\text { Umarzai }\end{array}$ \\
\hline \multirow[t]{4}{*}{3.} & \multicolumn{9}{|l|}{ Asteraceae } \\
\hline & Artimisia sp. & Tarkha & $\begin{array}{l}\text { Perennial } \\
\text { herb }\end{array}$ & $\begin{array}{l}\text { Wheat } \\
\text { field }\end{array}$ & $\begin{array}{l}\text { Cultivated } \\
\text { fields }\end{array}$ & $\begin{array}{l}\text { Whole } \\
\text { plant }\end{array}$ & $\begin{array}{l}\text { Diuretic, } \\
\text { febrifuge, } \\
\text { anodyne, and } \\
\text { anti- } \\
\text { inflammatory. }\end{array}$ & $\begin{array}{l}\text { May- } \\
\text { August }\end{array}$ & $\begin{array}{l}\text { Shabqadar, } \\
\text { Sherpao, Umarzai }\end{array}$ \\
\hline & Circium arvense (L.) Scop. & Azghakey & $\begin{array}{l}\text { Perennial } \\
\text { herb }\end{array}$ & $\begin{array}{l}\text { Wheat } \\
\text { field }\end{array}$ & $\begin{array}{l}\text { Disturbed } \\
\text { places }\end{array}$ & Leaves & $\begin{array}{l}\text { Fodder and } \\
\text { boiled water are } \\
\text { used for } \\
\text { dirrhoea }\end{array}$ & $\begin{array}{l}\text { May- } \\
\text { August }\end{array}$ & $\begin{array}{l}\text { Through out } \\
\text { Charsadda }\end{array}$ \\
\hline & $\begin{array}{l}\text { Parthenium hysterophorous } \\
\text { L. }\end{array}$ & $\begin{array}{l}\text { Zangley } \\
\text { tarkha }\end{array}$ & $\begin{array}{l}\text { Annual } \\
\text { herb }\end{array}$ & $\begin{array}{l}\text { Wheat } \\
\text { and } \\
\text { Garlic } \\
\text { field }\end{array}$ & $\begin{array}{l}\text { Disturbed } \\
\text { places }\end{array}$ & $\begin{array}{l}\text { Whole } \\
\text { plant }\end{array}$ & Fodder and fuel & $\begin{array}{l}\text { April- } \\
\text { October }\end{array}$ & $\begin{array}{l}\text { Through out } \\
\text { Charsadda }\end{array}$ \\
\hline
\end{tabular}




\begin{tabular}{|c|c|c|c|c|c|c|c|c|c|}
\hline & Sonchus oleraceous L. & Shodapay & $\begin{array}{l}\text { Annual } \\
\text { herb }\end{array}$ & $\begin{array}{l}\text { Wheat } \\
\text { field }\end{array}$ & $\begin{array}{l}\text { Cultivated } \\
\text { and } \\
\text { waste } \\
\text { fields }\end{array}$ & $\begin{array}{l}\text { Whole } \\
\text { plant }\end{array}$ & $\begin{array}{l}\text { Fodder, } \\
\text { febrifuge, } \\
\text { sedative, } \\
\text { diuretic } \\
\text { It also increase } \\
\text { milk production } \\
\text { in cattle }\end{array}$ & April-June & $\begin{array}{l}\text { Through out } \\
\text { Charsadda }\end{array}$ \\
\hline & Taraxacum officinale Weber. & Zyar guli & $\begin{array}{l}\text { Perennial } \\
\text { herb }\end{array}$ & $\begin{array}{l}\text { Wheat } \\
\text { field }\end{array}$ & $\begin{array}{l}\text { Waste } \\
\text { places }\end{array}$ & $\begin{array}{l}\text { Rhizome, } \\
\text { roots and } \\
\text { leaves }\end{array}$ & $\begin{array}{l}\text { Fodder, } \\
\text { constipation, } \\
\text { laxative, } \\
\text { diuretic, bitter } \\
\text { tonic, } \\
\text { vegetable. } \\
\text { Rhizomes are } \\
\text { used for coffee } \\
\text { preparation. }\end{array}$ & April-June & $\begin{array}{l}\text { Through out } \\
\text { Charsadda }\end{array}$ \\
\hline \multirow[t]{2}{*}{4.} & Boraginaceae & & & & & & & & \\
\hline & $\begin{array}{l}\text { Buglossoides arvensis (L.) } \\
\text { Johnston. }\end{array}$ & Not known & $\begin{array}{l}\text { Annual } \\
\text { herb }\end{array}$ & $\begin{array}{l}\text { Wheat } \\
\text { and } \\
\text { Garlic } \\
\text { field }\end{array}$ & $\begin{array}{l}\text { Cultivated } \\
\text { fields }\end{array}$ & Leaves & $\begin{array}{l}\text { Leaves are } \\
\text { used as diuretic }\end{array}$ & $\begin{array}{l}\text { March- } \\
\text { April }\end{array}$ & $\begin{array}{l}\text { Sherpao, Umarzai, } \\
\text { Tangi }\end{array}$ \\
\hline \multirow[t]{3}{*}{5.} & Brassicaceae & & & & & & & & \\
\hline & Brassica compestris L. & Sharsham & $\begin{array}{l}\text { Annual } \\
\text { herb }\end{array}$ & $\begin{array}{l}\text { Wheat } \\
\text { field }\end{array}$ & $\begin{array}{l}\text { Cultivated } \\
\text { fields }\end{array}$ & $\begin{array}{l}\text { Whole } \\
\text { plant }\end{array}$ & $\begin{array}{l}\text { Fodder, } \\
\text { vegetable oil } \\
\text { and salad }\end{array}$ & $\begin{array}{l}\text { March- } \\
\text { April }\end{array}$ & $\begin{array}{l}\text { Through out } \\
\text { Charsadda }\end{array}$ \\
\hline & Coronopus didymus (L.) Sm. & Sakha botay & $\begin{array}{l}\text { Annual } \\
\text { herb }\end{array}$ & $\begin{array}{l}\text { Wheat } \\
\text { and } \\
\text { Garlic } \\
\text { field }\end{array}$ & $\begin{array}{l}\text { Waste } \\
\text { places }\end{array}$ & $\begin{array}{l}\text { Leaves } \\
\text { and } \\
\text { shoots }\end{array}$ & $\begin{array}{l}\text { Fodder and } \\
\text { blood pressure }\end{array}$ & $\begin{array}{l}\text { May- } \\
\text { August }\end{array}$ & $\begin{array}{l}\text { Through out } \\
\text { Charsadda }\end{array}$ \\
\hline \multirow[t]{2}{*}{6.} & Cannabinaceae & & & & & & & & \\
\hline & Cannabis sativa L. & Bhang & $\begin{array}{l}\text { Perennial } \\
\text { herb }\end{array}$ & $\begin{array}{l}\text { Wheat, } \\
\text { Onion } \\
\text { and } \\
\text { Garlic } \\
\text { field }\end{array}$ & $\begin{array}{l}\text { Waste } \\
\text { places }\end{array}$ & $\begin{array}{l}\text { Leaves } \\
\text { and } \\
\text { flowering } \\
\text { buds }\end{array}$ & $\begin{array}{l}\text { Laxative, } \\
\text { sedative, } \\
\text { stimulant, } \\
\text { diuretic, } \\
\text { narcotics. }\end{array}$ & April-July & $\begin{array}{l}\text { Through out } \\
\text { Charsadda }\end{array}$ \\
\hline \multirow[t]{3}{*}{7.} & Caryophyllaceae & & & & & & & & \\
\hline & Cerastium glomeratum L. & Patewah & $\begin{array}{l}\text { Annual } \\
\text { herb }\end{array}$ & $\begin{array}{l}\text { Wheat } \\
\text { field }\end{array}$ & $\begin{array}{l}\text { Waste } \\
\text { places }\end{array}$ & $\begin{array}{l}\text { Whole } \\
\text { plant }\end{array}$ & $\begin{array}{l}\text { Juices are } \\
\text { obtained and } \\
\text { are used to } \\
\text { relieve } \\
\text { headaches. }\end{array}$ & April-July & $\begin{array}{l}\text { Shabqadar, } \\
\text { Umarzai, Tangi }\end{array}$ \\
\hline & Silene conoidea L. & Mangotai & $\begin{array}{l}\text { Annual } \\
\text { herb }\end{array}$ & $\begin{array}{l}\text { Wheat } \\
\text { field }\end{array}$ & $\begin{array}{l}\text { Dried } \\
\text { places }\end{array}$ & $\begin{array}{l}\text { Whole } \\
\text { plant }\end{array}$ & $\begin{array}{l}\text { Laxative and } \\
\text { purgative and } \\
\text { commonly used } \\
\text { as fodder }\end{array}$ & May-July & $\begin{array}{l}\text { Sherpao, Umarzai, } \\
\text { Tangi }\end{array}$ \\
\hline \multirow[t]{2}{*}{8.} & Chenopodiaceae & & & & & & & & \\
\hline & Chenopodium album $\mathrm{L}$. & $\begin{array}{l}\text { Zangali } \\
\text { gankhar }\end{array}$ & $\begin{array}{l}\text { Annual } \\
\text { herb }\end{array}$ & $\begin{array}{l}\text { Wheat } \\
\text { and } \\
\text { rice } \\
\text { field }\end{array}$ & $\begin{array}{l}\text { Waste } \\
\text { places }\end{array}$ & $\begin{array}{l}\text { Whole } \\
\text { plant }\end{array}$ & $\begin{array}{l}\text { Abdominal pain, } \\
\text { anthelminthic, } \\
\text { laxative, } \\
\text { vegetable and } \\
\text { fodder }\end{array}$ & $\begin{array}{l}\text { March- } \\
\text { May }\end{array}$ & $\begin{array}{l}\text { Through out } \\
\text { Charsadda }\end{array}$ \\
\hline \multirow[t]{2}{*}{9.} & Convululaceae & & & & & & & & \\
\hline & Convululus arvensis L. & Prewatai & $\begin{array}{l}\text { Climbing } \\
\text { herb }\end{array}$ & $\begin{array}{l}\text { Wheat } \\
\text { and } \\
\text { Garlic } \\
\text { field }\end{array}$ & $\begin{array}{l}\text { Cultivated } \\
\text { fields }\end{array}$ & $\begin{array}{l}\text { Whole } \\
\text { plant }\end{array}$ & $\begin{array}{l}\text { cancer and } \\
\text { stomach } \\
\text { disorder }\end{array}$ & $\begin{array}{l}\text { March- } \\
\text { April }\end{array}$ & $\begin{array}{l}\text { Through out } \\
\text { Charsadda }\end{array}$ \\
\hline \multirow[t]{2}{*}{10.} & Cuscutaceae & & & & & & & & \\
\hline & Cuscuta reflexa Roxb. & Banafsha & $\begin{array}{l}\text { Parasitic } \\
\text { weed }\end{array}$ & $\begin{array}{l}\text { Wheat } \\
\text { field }\end{array}$ & $\begin{array}{l}\text { Parasite } \\
\text { on other } \\
\text { plant }\end{array}$ & Shoots & $\begin{array}{l}\text { Diuretic, } \\
\text { anthelmintic, } \\
\text { jaundice, } \\
\text { vomiting and }\end{array}$ & April-June & $\begin{array}{l}\text { Sherpao, Umarzai, } \\
\text { Tangi }\end{array}$ \\
\hline
\end{tabular}




\begin{tabular}{|c|c|c|c|c|c|c|c|c|c|}
\hline & & & & & & & diabetic. & & \\
\hline 11. & Euphorbiaceae & & & & & & & & \\
\hline & Euphorbia heliscopia L. & Mandaroo & $\begin{array}{l}\text { Annual } \\
\text { herb }\end{array}$ & $\begin{array}{l}\text { Wheat, } \\
\text { Onion } \\
\text { and } \\
\text { Garlic } \\
\text { field }\end{array}$ & $\begin{array}{l}\text { Cultivated } \\
\text { field }\end{array}$ & $\begin{array}{l}\text { Root and } \\
\text { shoot }\end{array}$ & $\begin{array}{l}\text { Constipation, } \\
\text { anthelmintic, } \\
\text { cholera } \\
\text { Latex are used } \\
\text { for stoppage of } \\
\text { mences in } \\
\text { young female }\end{array}$ & April-July & $\begin{array}{l}\text { Through out } \\
\text { Charsadda }\end{array}$ \\
\hline 12. & Fabaceae & & & & & & & & \\
\hline & Lathyrus aphaca L. & Materr & $\begin{array}{l}\text { Annual } \\
\text { herb }\end{array}$ & $\begin{array}{l}\text { Wheat } \\
\text { field }\end{array}$ & $\begin{array}{l}\text { Waste } \\
\text { places }\end{array}$ & $\begin{array}{l}\text { Whole } \\
\text { plant }\end{array}$ & $\begin{array}{l}\text { Mostly used as } \\
\text { fodder } \\
\text { Seeds are } \\
\text { edible }\end{array}$ & $\begin{array}{l}\text { March- } \\
\text { April }\end{array}$ & $\begin{array}{l}\text { Shabqadar, } \\
\text { Sherpao }\end{array}$ \\
\hline & Medicago lupulina L. & $\begin{array}{l}\text { Peshtari } \\
\text { sagh }\end{array}$ & $\begin{array}{l}\text { Annual } \\
\text { herb }\end{array}$ & $\begin{array}{l}\text { Wheat } \\
\text { field }\end{array}$ & $\begin{array}{l}\text { Cultivated } \\
\text { fields }\end{array}$ & $\begin{array}{l}\text { Whole } \\
\text { plant }\end{array}$ & $\begin{array}{l}\text { Fodder, } \\
\text { vegetable and } \\
\text { for constipation }\end{array}$ & April-June & $\begin{array}{l}\text { Through out } \\
\text { Charsadda }\end{array}$ \\
\hline & Medicago polymorpha L. & $\begin{array}{l}\text { Peshtari } \\
\text { sagh }\end{array}$ & $\begin{array}{l}\text { Annual } \\
\text { herb }\end{array}$ & $\begin{array}{l}\text { Wheat } \\
\text { field }\end{array}$ & $\begin{array}{l}\text { Cultivated } \\
\text { fields }\end{array}$ & $\begin{array}{l}\text { Whole } \\
\text { plant }\end{array}$ & $\begin{array}{l}\text { Fodder, } \\
\text { vegetable and } \\
\text { leaves are used } \\
\text { for bitter taste }\end{array}$ & $\begin{array}{l}\text { March- } \\
\text { April }\end{array}$ & $\begin{array}{l}\text { Through out } \\
\text { Charsadda }\end{array}$ \\
\hline & Trifolium repens $\mathrm{L}$. & Shautal & $\begin{array}{l}\text { Perennial } \\
\text { herb }\end{array}$ & $\begin{array}{l}\text { Wheat } \\
\text { and } \\
\text { sugar } \\
\text { cane } \\
\text { field }\end{array}$ & $\begin{array}{l}\text { Cultivated } \\
\text { fields }\end{array}$ & $\begin{array}{l}\text { Whole } \\
\text { plant }\end{array}$ & $\begin{array}{l}\text { Fodder and } \\
\text { vegetable } \\
\text { The flowers are } \\
\text { used as honey } \\
\text { bee attractants }\end{array}$ & May-June & $\begin{array}{l}\text { Through out } \\
\text { Charsadda }\end{array}$ \\
\hline & Vicia sativa L. & Mater palli & $\begin{array}{l}\text { Annual } \\
\text { herb }\end{array}$ & $\begin{array}{l}\text { Wheat } \\
\text { field }\end{array}$ & $\begin{array}{l}\text { Cultivated } \\
\text { fields }\end{array}$ & $\begin{array}{l}\text { Whole } \\
\text { plant }\end{array}$ & $\begin{array}{l}\text { Fodder, seeds } \\
\text { are used for } \\
\text { bitter taste }\end{array}$ & $\begin{array}{l}\text { June- } \\
\text { August }\end{array}$ & $\begin{array}{l}\text { Shabqadar,Umarzai, } \\
\text { Tangi, Sherpao }\end{array}$ \\
\hline 13. & Fumariaceae & & & & & & & & \\
\hline & $\begin{array}{l}\text { Fumaria indica (Hausskn.) } \\
\text { H.N Pugsley }\end{array}$ & Papra & $\begin{array}{l}\text { Annual } \\
\text { herb }\end{array}$ & $\begin{array}{l}\text { Wheat } \\
\text { field }\end{array}$ & $\begin{array}{l}\text { Cultivated } \\
\text { fields }\end{array}$ & Shoots & $\begin{array}{l}\text { Blood purifier, } \\
\text { antipyretic, } \\
\text { whooping } \\
\text { cough, sore } \\
\text { throat } \\
\text { Commonly used } \\
\text { as fodder }\end{array}$ & April-June & $\begin{array}{l}\text { Through out } \\
\text { Charsadda }\end{array}$ \\
\hline 14. & Lamiaceae & & & & & & & & \\
\hline & Lamium amplixicule L. & Gulabi gulli & $\begin{array}{l}\text { Annual } \\
\text { herb }\end{array}$ & $\begin{array}{l}\text { Wheat } \\
\text { field }\end{array}$ & $\begin{array}{l}\text { Cultivated } \\
\text { fields }\end{array}$ & $\begin{array}{l}\text { Whole } \\
\text { plant }\end{array}$ & $\begin{array}{l}\text { Laxative and } \\
\text { stimulant }\end{array}$ & $\begin{array}{l}\text { March- } \\
\text { May }\end{array}$ & Shabqadar, Umarzai \\
\hline 15. & Oxilidaceae & & & & & & & & \\
\hline & Oxalis corniculata L. & $\begin{array}{l}\text { Qarghi } \\
\text { mewa }\end{array}$ & $\begin{array}{l}\text { Perennial } \\
\text { herb }\end{array}$ & $\begin{array}{l}\text { Wheat } \\
\text { and } \\
\text { Garlic } \\
\text { field }\end{array}$ & $\begin{array}{l}\text { Moist and } \\
\text { shady } \\
\text { places }\end{array}$ & $\begin{array}{l}\text { Leaves } \\
\text { and roots }\end{array}$ & $\begin{array}{l}\text { Fodder, } \\
\text { anthelmintic, } \\
\text { stomach pain } \\
\text { Leaves are } \\
\text { used for bitter } \\
\text { taste }\end{array}$ & April-June & $\begin{array}{l}\text { Through out } \\
\text { Charsadda }\end{array}$ \\
\hline 16. & Papaveraceae & & & & & & & & \\
\hline & Papaver dubium L. & Qashqash & $\begin{array}{l}\text { Annual } \\
\text { herb }\end{array}$ & $\begin{array}{l}\text { Wheat } \\
\text { field }\end{array}$ & $\begin{array}{l}\text { Cultivated } \\
\text { fields }\end{array}$ & $\begin{array}{l}\text { Seeds } \\
\text { and } \\
\text { capsule }\end{array}$ & $\begin{array}{l}\text { Narcotics and } \\
\text { purgative } \\
\text { The capsule are } \\
\text { used to make } \\
\text { tea taken orally } \\
\text { for cold and } \\
\text { cough }\end{array}$ & Feb-April & $\begin{array}{l}\text { Shabqadar, Tangi, } \\
\text { Sherpao }\end{array}$ \\
\hline 17. & Plantaginaceae & & & & & & & & \\
\hline & Plantago lenceolata L. & Asphaghol & $\begin{array}{l}\text { Perennial } \\
\text { herb }\end{array}$ & $\begin{array}{l}\text { Wheat } \\
\text { field }\end{array}$ & $\begin{array}{l}\text { Cultivated } \\
\text { fields and } \\
\text { waste } \\
\text { places }\end{array}$ & $\begin{array}{l}\text { Whole } \\
\text { plant }\end{array}$ & $\begin{array}{l}\text { Digestive, } \\
\text { constipation, } \\
\text { vomiting and } \\
\text { diarrhea }\end{array}$ & $\begin{array}{l}\text { March- } \\
\text { May }\end{array}$ & Shabqadar \\
\hline & Plantago major L. & Asphaghol & Perennial & Wheat & Cultivated & Leaves & Constipation & May-July & Shabqadar, Umarzai \\
\hline
\end{tabular}




\begin{tabular}{|c|c|c|c|c|c|c|c|c|c|}
\hline & & & herb & field & $\begin{array}{l}\text { fields and } \\
\text { waste } \\
\text { places }\end{array}$ & $\begin{array}{l}\text { and } \\
\text { seeds }\end{array}$ & and dysentery & & \\
\hline \multirow[t]{6}{*}{18.} & \multicolumn{9}{|l|}{ Poaceae } \\
\hline & Avena sativa L. & Jamdar & $\begin{array}{l}\text { Annual } \\
\text { herb }\end{array}$ & $\begin{array}{l}\text { Wheat } \\
\text { field }\end{array}$ & $\begin{array}{l}\text { Cultivated } \\
\text { fields }\end{array}$ & $\begin{array}{l}\text { Leaves, } \\
\text { stem and } \\
\text { seeds }\end{array}$ & $\begin{array}{l}\text { Commonly used } \\
\text { as fodder. } \\
\text { Antispasmodic, } \\
\text { nerve tonic. } \\
\text { Extract are } \\
\text { used for soothe } \\
\text { skin }\end{array}$ & $\begin{array}{l}\text { March- } \\
\text { June }\end{array}$ & $\begin{array}{l}\text { Through out } \\
\text { Charsadda }\end{array}$ \\
\hline & Cynodon dactylon (L.) Pers. & Kabal & $\begin{array}{l}\text { Rhizomitic } \\
\text { grass }\end{array}$ & $\begin{array}{l}\text { Wheat, } \\
\text { Onion, } \\
\text { Maize, } \\
\text { Rice } \\
\text { and } \\
\text { Sugar } \\
\text { cane } \\
\text { fields }\end{array}$ & $\begin{array}{l}\text { Every } \\
\text { where }\end{array}$ & $\begin{array}{l}\text { Whole } \\
\text { plant }\end{array}$ & $\begin{array}{l}\text { For blood } \\
\text { purifier, } \\
\text { jaundice and } \\
\text { dysentery }\end{array}$ & April-Sep & $\begin{array}{l}\text { Through out } \\
\text { Charsadda }\end{array}$ \\
\hline & Phalaris minor Retz. & Wakha & $\begin{array}{l}\text { Annual } \\
\text { grass }\end{array}$ & $\begin{array}{l}\text { Wheat } \\
\text { field }\end{array}$ & $\begin{array}{l}\text { Cultivated } \\
\text { and } \\
\text { waste } \\
\text { places }\end{array}$ & $\begin{array}{l}\text { Whole } \\
\text { plant }\end{array}$ & $\begin{array}{l}\text { Commonly used } \\
\text { as fodder }\end{array}$ & $\begin{array}{l}\text { March- } \\
\text { May }\end{array}$ & $\begin{array}{l}\text { Through out } \\
\text { Charsadda }\end{array}$ \\
\hline & Poa annua $\mathrm{L}$. & Wakha & $\begin{array}{l}\text { Annual } \\
\text { grass }\end{array}$ & $\begin{array}{l}\text { Wheat } \\
\text { field }\end{array}$ & $\begin{array}{l}\text { Cultivated } \\
\text { and } \\
\text { waste } \\
\text { places }\end{array}$ & $\begin{array}{l}\text { Whole } \\
\text { plant }\end{array}$ & $\begin{array}{l}\text { Fodder and } \\
\text { used for } \\
\text { cleaning of } \\
\text { utensils }\end{array}$ & $\begin{array}{l}\text { April- } \\
\text { October }\end{array}$ & $\begin{array}{l}\text { Through out } \\
\text { Charsadda }\end{array}$ \\
\hline & $\begin{array}{l}\text { Rostraria cristata (L.) } \\
\text { Tzvelev. }\end{array}$ & Narri wakha & $\begin{array}{l}\text { Annual } \\
\text { grass }\end{array}$ & $\begin{array}{l}\text { Wheat } \\
\text { field }\end{array}$ & $\begin{array}{l}\text { Cultivated } \\
\text { and } \\
\text { waste } \\
\text { places }\end{array}$ & $\begin{array}{l}\text { Whole } \\
\text { plant }\end{array}$ & $\begin{array}{l}\text { Grazed by } \\
\text { animal as } \\
\text { fodder }\end{array}$ & April-July & $\begin{array}{l}\text { Through out } \\
\text { Charsadda }\end{array}$ \\
\hline \multirow[t]{2}{*}{19.} & \multicolumn{9}{|l|}{ Polygonaceae } \\
\hline & Rumex hestatus D.Don & Shalkhay & $\begin{array}{l}\text { Annual } \\
\text { herb }\end{array}$ & $\begin{array}{l}\text { Wheat } \\
\text { and } \\
\text { sugar } \\
\text { cane } \\
\text { field }\end{array}$ & $\begin{array}{l}\text { Cultivated } \\
\text { fields and } \\
\text { waste } \\
\text { places }\end{array}$ & Leaves & $\begin{array}{l}\text { Astringic, } \\
\text { diuretic and } \\
\text { stomachic. } \\
\text { Leaves are } \\
\text { used as cattle } \\
\text { fodder }\end{array}$ & April-June & $\begin{array}{l}\text { Through out } \\
\text { Charsadda }\end{array}$ \\
\hline \multirow[t]{4}{*}{20.} & \multicolumn{9}{|l|}{ Ranunculaceae } \\
\hline & Adonis aestivalis $\mathrm{L}$. & Mekhaki & $\begin{array}{l}\text { Annual } \\
\text { herb }\end{array}$ & $\begin{array}{l}\text { Wheat } \\
\text { field }\end{array}$ & $\begin{array}{l}\text { Moist } \\
\text { places }\end{array}$ & Flowers & $\begin{array}{l}\text { Diuretic, cardio } \\
\text { tonic, laxative } \\
\text { and stimulant }\end{array}$ & $\begin{array}{l}\text { March- } \\
\text { June }\end{array}$ & $\begin{array}{l}\text { Umarzai, Sherpao, } \\
\text { Tangi }\end{array}$ \\
\hline & Ranunculus arvensis L. & Zyar gulay & $\begin{array}{l}\text { Annual } \\
\text { herb }\end{array}$ & $\begin{array}{l}\text { Wheat } \\
\text { field }\end{array}$ & $\begin{array}{l}\text { Moist } \\
\text { places }\end{array}$ & $\begin{array}{l}\text { Whole } \\
\text { plant }\end{array}$ & $\begin{array}{l}\text { Grazed by } \\
\text { animals as } \\
\text { fodder }\end{array}$ & May-July & $\begin{array}{l}\text { Umarzai, Tangi, } \\
\text { Shabqadar }\end{array}$ \\
\hline & Ranunculus muricatus $\mathrm{L}$. & Zyar gulay & $\begin{array}{l}\text { Annual } \\
\text { herb }\end{array}$ & $\begin{array}{l}\text { Wheat } \\
\text { field }\end{array}$ & $\begin{array}{l}\text { Moist } \\
\text { places }\end{array}$ & $\begin{array}{l}\text { Whole } \\
\text { plant }\end{array}$ & $\begin{array}{l}\text { Toxic and } \\
\text { laxative } \\
\text { Mostly used as } \\
\text { fodder }\end{array}$ & April-June & $\begin{array}{l}\text { Umarzai, Sherpao, } \\
\text { Tangi }\end{array}$ \\
\hline \multirow[t]{2}{*}{21.} & \multicolumn{9}{|l|}{ Solanaceae } \\
\hline & Solanum nigrum L. & kachmachoo & $\begin{array}{l}\text { Annual } \\
\text { herb }\end{array}$ & $\begin{array}{l}\text { Maize } \\
\text { and } \\
\text { sugar } \\
\text { cane } \\
\text { field }\end{array}$ & $\begin{array}{l}\text { Waste } \\
\text { places }\end{array}$ & $\begin{array}{l}\text { Fruits, } \\
\text { leaves } \\
\text { and stem }\end{array}$ & $\begin{array}{l}\text { Inflammation, } \\
\text { dysentery and } \\
\text { for low blood } \\
\text { pressure }\end{array}$ & April-June & $\begin{array}{l}\text { Through out } \\
\text { Charsadda }\end{array}$ \\
\hline
\end{tabular}


Table 5: Summary of percentage of the plant parts used for traditional medicine and economic importance.

\begin{tabular}{cccc}
\hline S.No. & Parts used & $\begin{array}{c}\text { No. of } \\
\text { species }\end{array}$ & Percentage $\%$ \\
\hline 1. & Whole plant & 24 & $60 \%$ \\
\hline 2. & Leaves & 3 & $7.5 \%$ \\
\hline 3. & $\begin{array}{c}\text { Leaves, stem and } \\
\text { seeds }\end{array}$ & 2 & $5 \%$ \\
\hline 4. & Shoot & 2 & $5 \%$ \\
\hline 5. & Flower & 1 & $2.5 \%$ \\
\hline 6. & $\begin{array}{c}\text { Rhizome, roots } \\
\text { and leaves }\end{array}$ & 1 & $2.5 \%$ \\
\hline 7. & Leaves and shoots & 1 & $2.5 \%$ \\
\hline 8. & $\begin{array}{c}\text { Leaves and } \\
\text { flowering tops }\end{array}$ & 1 & $2.5 \%$ \\
\hline 9. & Leaves and roots & 1 & $2.5 \%$ \\
\hline 10. & Leaves and seeds & 1 & $2.5 \%$ \\
\hline 11. & $\begin{array}{c}\text { Leaves, fruits and } \\
\text { stem }\end{array}$ & 1 & $2.5 \%$ \\
\hline 12. & Roots and shoots & 1 & $2.5 \%$ \\
\hline 13. & $\begin{array}{c}\text { Seeds and } \\
\text { capsule }\end{array}$ & 1 & $2.5 \%$ \\
\hline & Total & 40 & $100 \%$ \\
\hline
\end{tabular}

The percentage of the part used of economically important plant species are; whole plant (24 species, $60 \%$ ), leaves, stem and seeds ( 2 species, $5 \%$ ), leaves ( 3 species, $7.5 \%$ ), shoots (2 species, $5 \%$ ), rhizome, roots and leaves ( 1 specie, $2.5 \%$ ), leaves and shoots (1 specie, $2.5 \%$ ), leaves and flowering buds (1 specie, $2.5 \%$ ), flowers (1 specie, 2.5\%), roots and shoots (1 specie, $2.5 \%$ ), leaves and roots (1 specie, $2.5 \%$ ), seeds and capsule (1 specie, $2.5 \%$ ), leaves and seed (1 specie, $2.5 \%$ ), fruits, leaves and stem (1 specie, $2.5 \%$ ) are shown in Table 5. These plants species were collected from different crops field viz wheat, Maize and sugar cane fields. Some economically weed species are growing on cultivated and waste fields some were growing on waste, disturbed, dried, moist and shady places and some are parasite on other plants. The plants which are used for the treatment of various ailments are toothache, cough, asthma, removal of kidney stone, anti-inflammatory, diarrhoea, increase milk production in cattle, constipation, relive headache, anthelminthic, anti-cancer, stomach disorder , vomiting, jaundice, diabetic, cholera, whooping cough, blood purifier, sore throat, laxative, stimulant, diuretic, stomach pain, narcotics, purgative, digestive, nerve tonic, dysentery, antispasmodic, astringic, cardio tonic, stimulant, low blood pressure and inflammation and some are economically importance and used for different purposes i-e vegetable, vegetable oil, fuel, coffee, salad, edible, bitter tonic, insect attractants, cleaning of utensils and mostly are used as fodder. The local people of Charsadda have been totally depend on plant resources for their various ailments since time to time specially poor people. The inhabitants know about the preparation of the useful plants through long experience. Charsadda is very large and therefore local inhabitants of the area are totally depending on natural resources as compared to urban people. The present work showed that local inhabitants of the area are most familiar and rich in indigenous knowledge of plant resources also their ethno botanical and ethno medicinal uses to treat for various purposes. Several hakims (local healers) famous not only locally but also the people from other parts of the country visit them for treatment. Many of them do not charge anything for their service that guide the people and treat them without any payment ${ }^{6}$. Traditional uses of plant help ecologists, pharmacologists, taxonomists, watershed and wild life managers in their efforts for improving the wealth of rea $^{8}$. Many scientists carried out various ethno botanical researches as some of prominent are; Jan et al. elaborate the indigenous uses of 26 ethno botanically important weeds belonging to 16 families from common weeds of Dir, Kohistan Valley, Khyber Pakhtoonkhwa, Pakistan ${ }^{17}$. Razzaq et al. studied and documented total of 50 taxa, belonging to 32 families are used for various purposes to elaborate the ethno medicinal plant potential of Changa valley district Shangla, Pakistan ${ }^{18}$. Farooq et al. screened out 72 plant species from tehsil Birmal of South Waziristan Agency included 49 medicinal plants, 16 forage, 11 fuel wood, nine vegetables, six agriculture tools, six ornamentals, four nectariferous, three tonic, two each for fencing and spices and one each for timber, furniture, confectionary and desserts, narcotic, cosmetic and insect repellent ${ }^{18}$. Local community was using 58 plant species belonging to 52 genera and 34 families for different purposes from Banda Daud Shah, District Karak, Pakistan ${ }^{20}$. According to $\mathrm{Naz}$ et al. 80 plant species belonging to 64 genera and 36 families used by local 
inhabitants for the treatment of various ailments. Hadi and his co-worker explore the ethno botanical uses of weeds were also investigated ${ }^{22}$. For this purpose local 15 males and 10 females were interviewed about the indigenous use of these weeds. About 200 local inhabitants were found that utilizing 52 plants of 37 families for various ethno botanical purposes, such as timber, fodder and forage ${ }^{23}$. Fazalullah et al. also conducted the same study as 40 weeds species were observed from the wheat crop belonging to 21 families and 38 genera for their medicinal values and ecological importance from lower $\mathrm{Dir}^{24}$. According to Prabhu \& Vijayakumar, 27 species of plants distributed in 27 genera belonging to 18 families were identified as commonly used ethno medicinal plants by ethnic people. Wani et al. estimated 27 threatened plants along with their ethno botanical uses from District Baramulla, Kashmir, Jammu and Kashmir, India ${ }^{26}$. Jan et al. also investigated the same ethnobotanical study from Tehsil Tangi, District Charsadda27. 38 families were reported in which 4 families were monocot and 34 families were dicot. Among them 50 species were medicinally treated for various diseases in which (6 spp) were used for Diarrhea, Dysentery and pain killer, (4 spp) used for Asthma and diabetes, (3 spp) were used as anti-septic and (2 spp) as a Laxative. This work is the first ever report on economic importance of the weed flora of district Charsadda, KP, Pakistan which might help for future intensive and extensive researcher.

\section{ACKNOWLEDGEMENT}

I am grateful to my honorable Teacher Sir Ghulam Jelani for plants identification and providing me research facilities throughout the whole period of this study. I want to pay great thanks and I am feeling honorable to $\mathrm{Dr}$. Abdul Razzaq and Dr. Fazal Hadi for his sincere cooperation, for his generosity, great suggestions, for his attitude and awesome support.

\section{REFERENCES}

1. Anonymous (1998). District Census Report of Charsadda. PCO, Govt. of Pakistan.

2. Khan SM, Din NU, llyas M, Sohail UR, ljaz F, lqbal Z, Ali $Z$. Ethnobotanical study of some medicinal plants of Tehsil Kabal, District Swat, KP, Pakistan. Med Aromat Plants., 2015; 4: 189.
3. Jabeen, T. and S.S. Ahmed. Multivariate analysis of environmental and vegetation data of Ayub National Park Rawalpindi. Soil Environ. 2009; 28(2): 103-12.

4. Sher ZF, Hussain L, Badshah M, Wahab M. Floristic composition, communities and ecological characteristics of weeds of wheat fields of Lahore, District Swabi, Pakistan. Pak J Bot. 2011; 43(6): 2817-20.

5. Ali S I. The significance of flora with special reference to Pakistan. Pak J Bot. 2008; 40(30): 967-71.

6. Qureshi RU, Gilani SA, Ghufran MA. Ethnobotanical studies of plants of Mianwali District Punjab, Pakistan. Pak J Bot. 2007; 39(7): 2285-90.

7. Bhushan B, Kumar M. Ethnobotanically important medicinal plants of Tehsil Billawar, District Kathua, J\&K. Ind J Pharmacog Phytochem. 2013; 2 (4): 14-21.

8. Ibrar M, Hussain F, Sultan A. Ethnobotanical studies on plant resources of Ranyal Hills, District Shangla, Pakistan. Pak J Bot. 2007; 39(2): 329-37.

9. Sher Z, Khan ZU, Hussain F. Ethnobotanical studies of some plants of Chagharzai Valley, District Buner, Pakistan. Pak J Bot. 2011; 43(3): 1445-2.

10. Ali M, Begum R, Ali K, Nisar M, Khan W, Hassan N, et al. Ethnobotanical survey of wild medicinal plants of Tirat Valley District Swat, Khyber-Pakhtunkhwa. J Biodiver Environ Sci. 2017; 11(3): 91-101.

11. Pandey D, Pandey VC. Sacred plants from ancient to modern era: Traditional worshipping towards plants conservation. Tropical Plant Research 2016; 3(1): 13641.

12. Mengesha GG. Ethnobotanical survey of medicinal plants used in treating human and livestock health problems in Mandura Woreda of Benishangul Gumuz, Ethiopia. Adv Med Plant Res. 2016; 4(1): 11-26.

13. Ullah ZR, Ullah G, M. Shah A, Majeed M, Hussain M, H Ullah. Ethnomedicinal plants of district Charsadda Khyber Pakhtunkhwa, Pakistan. J Biodivers Environ Sci. 2016; 9(2): 254-64.

14. Ali SI, Nasir Y (1971-1991). Flora of Pakistan. Department of Botany, University of Karachi.

15. Nasir Y, Ali SI (1991-1993). Flora of Pakistan. Department of Botany, University of Karachi.

16. Ali SI, Qaiser M (1995-2018). Flora of Pakistan. Department of Botany, University of Karachi.

17. Jan G, Khan MA, Gul F, Ahmad M, Jan M, Zafar M. Ethno botanical study of common weeds of Dir Kohistan Valley, Khyber Pakhtoonkhwa, Pakistan. Pak J Weed Sci Res. 2010; 16(1): 81-8.

18. Razzaq A, Rashid A, Ali H, Ahmad H, Islam M. Ethnomedicinal potential of plants of Changa Valley, Distt; Shangla. Pak J Bot. 2010; 42(5): 3463-75.

19. Farooq S, Barki AY, Khan MY, Fazal H. Ethnobotanical studies of the flora of Tehsil Birmal in South Waziristan Agency, Pakistan. Pak J Weed Sci Res. 2012; 18(3): 277-91.

20. Murad W, Ullah AUA, Adnan M, Tariq A, Khan KU, Waheed S, Ahmad A. Ethnobotanical assessment of 
plant resources of Banda Daud Shah, District Karak, Pakistan. J Ethnobiol Ethnomed. 2013; 9:77.

21. Naz I, Ahmad M, Hassan TU. Ethnobotanical investigation of medicinal flora used by indigenous people in District Attock, Pakistan. J Advan Bot Zool 2014; 1(4):1-7.

22. Hadi F, Rahman AU, Ibrar M, Dastagir G, Arif M, Naveed $\mathrm{K}$, et al. Weed diversity in wheat and maize with special reference to their ethnomedicinal uses at Rech valley, Hindokush Range, Chitral, Pakistan. Pak J Weed Sci Res. 2014; 20(3): 335-46.

23. Hassan HU, Murad W, Ahmad N, Tariq A, Khan I, Akhtar $\mathrm{N}$, et al. Indigenous uses of the plants of Malakand valley, district Dir (Lower), Khyber Pakhtunkhwa, Pakistan. Pak J Weed Sci Res. 2015; 21(1): 83-99.
24. Ullah F, Ullah A, Sohail A. Medicinal and ecological diversity of weeds in wheat crop at Lower Dir, Pakistan. Pak J Weed Sci Res. 2016; 22(4): 627-637.

25. Prabhu S, Vijayakumar S. Ethnobotanical study of traditionally used medicinal plants in Malayali ethnic people of Pachamalai hills, Tamil Nadu, India. J Pharm Med Res. 2016; 2(1) 39-42.

26. Wani Z, Kumar A, Akash N. Ethnobotanical study of some threatened plants in District Baramulla, Kashmir, Jammu and Kashmir, India. Int J Curr Res Biosci Plant Biol. 2016; 3(2), 58-64.

27. Jan RU, Khan RU, Rehman HU, Khan AZ, Waheed MA, Khan IU, et al. Ethnobotanically important flora of Tehsil Tangi, District Charsadda, Pakistan. J Chem Pharmaceu Res. 2016; 8(3):108-16. 